\title{
Erratum to: The Efficacy of Process Capability Indices Using Median Absolute Deviation and Their Bootstrap Confidence Intervals
}

\author{
Muhammad Kashif $^{1}$ - Muhammad Aslam ${ }^{1}(\mathbb{D})$ Chi-Hyuck Jun ${ }^{2}$ • \\ Ali Hussein Al-Marshadi ${ }^{1}$. G. Srinivasa Rao ${ }^{3}$
}

Published online: 7 September 2017

(C) King Fahd University of Petroleum \& Minerals 2017

\section{Erratum to: Arab J Sci Eng \\ DOI 10.1007/s13369-017-2699-4}

The original version of this article unfortunately contained a mistake. The spelling of the fourth author's name was incorrect. The correct name is given here.

The original article has been corrected.

The online version of the original article can be found under doi:10.1007/s13369-017-2699-4.

Muhammad Aslam

aslam_ravian@hotmail.com

Muhammad Kashif

mkashif@uaf.edu.pk

Chi-Hyuck Jun

chjun@postech.ac.kr

Ali Hussein Al-Marshadi

aalmarshadi@kau.edu.sa

G. Srinivasa Rao

gaddesrao@gmail.com

1 Department of Statistics, Faculty of Sciences, King Abdulaziz University, Jeddah 21551, Saudi Arabia

2 Department of Industrial and Management Engineering, POSTECH, Pohang 37673, Republic of Korea

3 Department of Statistics, School of Mathematical Sciences, CNMS, The University of Dodoma, PO Box 259, Dodoma, Tanzania 